\title{
Data Analysis for Synchrotron Microcrystal Native-SAD Phasing
}

Qun Liua, , Gongrui Guo a,b, Ping Zhua, Martin R. Fuchs ${ }^{b}$, Wuxian Shib, Babak Andib, Yuan Gao ${ }^{b}$, Wayne A. Hendrickson ${ }^{\mathrm{c}, \mathrm{d}}$, Sean McSweeney ${ }^{\mathrm{b}}$

a Biology Department, Brookhaven National Laboratory, Upton, NY 11973, USA

${ }^{b}$ Photon Science, NSLS-II, Brookhaven National Laboratory, Upton, NY 11973, USA

c Department of Biochemistry and Molecular Biophysics, Columbia University, New York, NY 10032, USA.

${ }^{d}$ Department of Physiology and Cellular Biophysics, Columbia University, New York, NY 10032, USA.

Contact email: qunliu@bnl.gov

De novo structural evaluation of native biomolecules from single-wavelength anomalous diffraction (SAD) is a challenge because of the weakness of the anomalous scattering. For microcrystals with sizes less than $10 \mu \mathrm{m}$, native-SAD phasing at synchrotron microdiffraction beamlines is even more challenging due to difficulties in sample manipulation, diffraction data collection and data analysis. Native-SAD analysis from microcrystals by using X-ray free electron lasers has been demonstrated but has required use of thousands of thousands of microcrystals to achieve the necessary accuracy. Here we show that by exploitation of anomalous microdiffraction signals at a synchrotron beamline, by the use of polyimide wellmounts, and by an iterative crystal and frame rejection method, optimized data may be acquired and assembled which allows microcrystal native-SAD phasing from as few as about 1200 crystals. Beyond microcrystals, our data analysis strategy may work equally well for microdiffraction data from larger crystals. 